\title{
PERSONEN- UND ORTSREGISTER
}

\section{Hinweise zum Register}

Paris, Berlin und London wurden ebenso wie Preußen-Deutschland, Großbritannien/England und Frankreich nicht als Ortsbegriffe in das Register übernommen. Für die Hauptstädte sind stattdessen Straßen und Plätze etc. direkt aufgefuihrt.

Seitenangaben unmittelbar nach Personennamen beziehen sich auf Textstellen, die die entsprechenden Personen nicht primär als Denkmalfigur erwähnen. Den Personennamen folgen Belegstellen zu den jeweiligen Denkmälern bzw. Projekten einschließlich des Jahres ihrer Einweihung bzw. Initiierung. Anders als der chronologische Anhang erfaßt das Register auch Denkmäler und Projekte, die nach den in Kap. I,2.2.3 dargelegten Kriterien analytisch nicht eingehender berücksichtigt wurden. Fehlen Vornamen, so waren sie nicht ermittelbar. Bauwerke werden aufgeführt, wenn sie in der Darstellung (auch) als Ortsangaben firmieren.

Abbeville 558, 560

Aberdeen, George Gordon, Gf. v. 229

Abgeordnetenhaus 49lf.

Achenbach, Heinrich v. 93

Admirality Arch 412

Akers-Douglas, Aretas 101, 104

Albert, Alexandre-Martin 297

Albert, Prince Consort 172f., 174f., 181, $183 f$., $188,253,359,405 f$., 416, 524, 652,683

- Dkm. (1863) 173f., 176-179, 188

- Dkm. (1874) 179

- Dkm. (1876) 99f., 118, 179-183, 188f., 412,423

Aldergate-Street 137

Alembert, Jean Le Rond d' 288

Alexander I., russischer Zar 150

Alexander II., russischer Zar 150

Alexanderplatz 92

Alexandra, Königin v. England

- Dkm. (1908) 652-654

Alfred, König v. England

- Dkm. in Winchester $v$. Thornycroft (1901/2) 324f.

Alphand, Jean-Charles Adolphe 291

Alpy 351

Alsenstraße 454

Annam 451

Anne, Königin v. England

- Dkm. (1712) 405 Anm. 323

- Dkm. (1886) 651, 654

Arago, François 245, 302, 584

- Dkm. (1893) 295, 301f.

Argyll, George Douglas Campbell,

$8^{\text {th }}$ Duke of 225
Amold, Thomas

- Büste in Westminster Abbey (1896) 570, 572, 576

Askanischer Platz 506f

Asquith, Herbert 419, 422, 517

Augsburg 113

August Wilhelm, Prinzessin (Alexandra Victoria) 661

Augusta, dt. Kaiserin, Gemahlin Wilhelms I.

- Dkm. (1895) 650f., 654

Auguste Viktoria, dt. Kaiserin, Gemahlin Wilhelms II. 594, 603

Avenue Clichy 559

Avenue de l'Observatoire 191

Avenue de la République 349

Avenue de Neuilly 153

Avenue Ledru-Rollin 283

Avenue Messine 609

Avenue Montaigne 150

Avenue Parmentier 296

Balfour, Arthur James 101, 103, 319 , $416,421,510,668$

Ballestrem, Franz v. 398

Balzac, Honoré de

- Dkm. (1902, Av. de Friedland) 610

Barrot, Ferdinand 192

Bartholdi, Frédéric Auguste 289

Bastide, Jules 158

Baudin, Alphonse 157, 283, 337, 683

- Dkm.-projekt (1868) 157-160, 189f., $238,274,281,337,344,683,698$

- Dkm. (1872) 281, 332, 682

- Dkm. (1901) 283-285, 332, 337, 682 
- Pantheonisierung (1889) 283, 289

Bavoux, Évariste 544

Beauharnais, Eugène de

- Dkm. (1863) 105, 149, 187

Beauharnais, Joséphine de, Gemahlin Napoleons I. 150

- Dkm. (1867) 149f., 185, 187, 697

Bebel, August 305, 310

Bebelplatz 204

Beckedorff, Georg Philipp v. 247

Begas, Reinhold 89

Behnes, William 213

Belfast 319

Belle Alliance s. Waterloo

Belle Alliance-Platz 487

Bentinck, George $219 f$.

- Dkm. (1851) 137, 145

Béranger, Jean

- Dkm. (1885) 481, 537

Berger, Georges 110

Berliner Rathaus 27

Bernard, Claude

- Dkm. (1886) 108, 583

Bersier, Eugène 556

Bert, Paul 583

Berthelot, Marcelin

- Dkm. (1917) 657f.

Bertrand, Joseph L.F. 584

Bethmann-Hollweg, Theobald v. 88, 661f.

Beuth, Peter Christian 249f.

- Dkm. (1861) 247, 249-252, 256-259, 675,694

Biez, Jacques de 546

Binder, Maurice 282

Bismarck, Herbert 398

Bismarck, Otto v. 28, 87, 355, 384, 396, $441,444 f$., 454

- Denkmäler 371, 397, 685

- Dkm. (1897) 398

- Dkm. (1901) 113, 398-404, 455f., $661-663,685$

- Dkm. (1904) 403f.

Bissinger, Herrmann 595

Blanc, Louis 106, 300-303

- Dkm. (1887) 295f., 299, 338f.

Blanqui, Auguste

- Grabdkm. (1885) 280

Blomfield, Charles James, Bf. v. London 631

Blondel, Jean 267

Bloomsbury Square 138

Blücher, Gebhard Leberecht v., Fst. v.
Wahlstatt

- Dkm. (1826) 91, 168, 204f

Bobilot, Jules

- Dkm. (1888) 449f., 468, 471, 687

Böhm, v. 661

Bois de Boulogne 246

Bonaparte s. Napoleon I.

Bonaparte, Louis-Napoléon s. Napoleon III.

Borthwick, Algernon 407f.

Borussia 455

Boulanger, Georges $347,349,430 f$., 449 , 682

Boulevard de Clichy 613

Boulevard de l'Impératrice Joséphine (i.e. Avenue Marceau) 149

Boulevard des Batignolles 196

Boulevard Edgar-Quinet 301

Boulevard Port-Royal 580

Boulevard Prince-Eugène-de-Beauharnais 149

Boulevard Raspail 301

Boulevard Richard-Lenoir 107, 348f., 366,449

Boulevard Saint-Germain 293, 554

Boulevard Saint-Martin 366

Boulevard Saint-Michel 547

Boulevard Voltaire 296, 366

Bourgeois, Léon 658

Bourneville, Désiré 555

Bousset, H. 385-387

Boutroux, Émile 657

Bradlaugh, Charles 215

Brandenburg 390, 392, 453f., 560f

Brandenburger Tor $165,197,372,379$, $394,505 f$, $592,620,648,650$

Breslau 131

Briand, Aristide 352, 354f., 645

Brisson, Henri 477

Britannia 41, 45, 51

Brock, Thomas 101, 414

Brompton Oratory 569

Brompton Road 465

Brougham, Henry 631

Brousse, Paul 644

Buckingham Palace 100, 209, $411 \mathrm{f}$., 414, $417,422,425,435$

Bülow, Bemhard v. 398, 400f.

Bülow, Friedrich Wilhelm, Gf. v.

- Dkm. (1822) 91, 168, 204

Burdach, Konrad 624

Burgoyne, John, Sir 
- Dkm. (1877) 228, 464, 667

Burgund 444

Burns, Robert

- Dkm. (1884) 118, 627, 635-638, 641, 696

Buzenval 439

Byron, George, Lord

- Dkm. (1880) 627, 630-632, 638, 641, 696f.

Calvin, Johannes

- Dkm. (1885) 560-562

Cambridge, Prince George, Duke of 224, 231, 410

- Dkm. (1907) 100, 466-469, 472f., 688

Camden High Street 140

Campbell, Colin, Sir (Baron Clyde)

- Dkm. (1867) 224, 228, 234, 237, 686

Campbell-Bannerman, Henry, Sir 101

Canning, George 17, 136, 138

- Dkm. (1832, New Palace Yard) 138, 145

Canterbury, Edward W. Benson, Erzbf. v. $514 \mathrm{f}$.

Cardwell, Edward, $1^{\text {st }}$ Viscount 138

Carlton Gardens 228

Carlyle, Thomas $45,633 f$.

- Dkm. (1882) 627, 633-635, 641, 696f.

Carnot, Adolphe 352

Carnot, Sadi 107, 343

Cavaignac, Godefroy 126, 157

Cavell, Edith 659

- Dkm. (1920 Jardin des Tuileries) 659f., 698

- Dkm. (1920 St. Martin's Place) 667f., 698

Cavendish Square 137

Celle 247

Challis, Thomas 173f.

Chamberlain, Austen 319, 416, 466, 511

Chamberlain, Joseph 101

Chamisso, Adalbert v.

- Dkm. (1888) 623f., 639, 696

Champs Elysées 358, 610, 629

Charing Cross $311,327-330$

Charité-Straße 505

Charlemagne/Karl der Große

- Dkm.-projekte (1863f.) 148

Charles I., König v. England und Schottland

- Dkm. (1676, Charing Cross) 311f., 314f., 327-330, 340, 405 Anm. 323
Charles VII., Kg. v. Frankreich 534

Charlottenbrücke 393

Charlottenburger Chaussee 595

Chaumié, Joseph 367

Cheapside 137

Chelsea Embankment 633

Cherbourg 153

Chevalier de la Barre 558

- Dkm. (1906) 559

Cimetière de Montmartre 157, 159, 281, 283

Cimetière du Montparnasse 301

Cimetière du Nord 282

Cimetière du Père Lachaise s. Père Lachaise

City Road Chapel 567

Clemenceau, Georges 348-350, 476-480, 548,645

Clésinger, Auguste 375

Clive, Robert, Baron

- Dkm. (1912) 226

Clyde, Lord

- Dkm. (1867) 464, 667

Cobden, Richard 141f, 513

- Dkm. (1868) 97, 140-142, 145f.

Cochin, Denys 29lf., 446

Cochinchina 451

Colas, Albert 645

Cole, Henry 182

Coligny, Gaspard de

- Dkm. (1889) 109f., 289, 554, 556f., 572, 576f., 691

Collège de France 108, 657

Conservatoire des Arts et Métiers 581

Cook, James

- Dkm. (1914) 117, 601-603, 607, 667

Coppée, François

- Dkm. (1910) 482f., 537, 587

Cour d'Honneur des Invalides 270

Cour du Louvre 128

Courbet, Gustave 268

Courbevoie 153, 269, 440, 442

Cowper, Francis William 139

Crawford, John Gordon 118, 635

Crémieux, Adolphe 281

Crimea Guards Memorial 255, 464, 665

Cromwell, Oliver 261, 293, 311-314, $316-318,322-324,461,635,681$

- Dkm.-projekt (1845) 311-315, 319

- Dkm. (1875, Manchester) 315

- Dkm. (1899) 103f., 261, 311, 315-326, 328f., $333,339,574,690,698$ 
Crystal Palace 173

Cubitt, William 98, 180

Dalhousie, James A. Broun-Ramsay, $1^{\text {st }}$ Marquess of 223

Dalou, Jules 289, 432

Danton, Georges 294

- Dkm. (1891) 106, 285, 288, 292-294, 337

Daubenton, Louis

- Dkm. (1864) 246

Daumas 553

Daumesnil, Pierre

- Dkm. (1873, Vincennes) 197

Dausset, Louis 283-285

Dautresme, Lucien 581

»Défense de la Barrière de Clichy« (1869) 196

》Défense de Paris « (1883) 84f., 106, 440-442f., 468, 470, 687

Delabrousse, Lucien 276

Delafosse, Jules $352 \mathrm{f}$.

Delbrück, Rudolf 252

Delescluze, Louis Charles 157-160

Delhomme, Léon 554

Derby, Earl of $62,175,520,522$

- Dkm. (1874) 10lf., 116, 140, 519, 521, 532, 538f., 690

Déroulède, Paul 364, 353, 362, 365, 483, 548

Derré, Emile 643

Desmoulin, L. 284f.

Desplas 586

Dessoye, Arthur 354

Deutscher Michel 31

Devonshire, Duke of (Spencer Compton

Cavendish) 511f.

- Dkm. (1911) 536

Diderot, Denis

- Dkm. (1886) 285f., 288, 30l, 332

Dijon 444

Disraeli, Benjamin $62,78,139,142,175$, $325,457,459,461,510,520-522,525-$ $529,532,632$

- Dkm. (1883) 102f., 327, 523-525, 529, 538f., 690

Dolet, Étienne

- Dkm. (1889) 109, 289, 554-556, 572, 575

Dom, Berlin 27, 205

Domrémy 545

Dönhoffplatz 372, 491, 496, 592
Doulton, Henry, Sir 514

Dreyfus, Alfred $341,353,365-368,475$ 480, 484f., 612, 688f.

Du Bois Reymond, Emil 1 14, 591

Dublin 101, 212, 322

Dubost, Antonin 476f.

Duc d'Orléans, Ferdinand-Philippe 128

Dumas, Alexandre

- Dkm. (1883) 106,609

Duncker, Hermann 616f., 620

Duncombe, Thomas S. 253

Dupanloup, Bf. v. Orléans 552

Dupin, André 194, 241

Düsseldorf 113

Duval, Ferdinand 439

East End 652f.

École nationale des Beaux-Arts 439

Edinburgh 101, 212

Edward VII., Prince of Wales und (ab 1911) Kg. v. Großbritannien 100f., 117, $321,326,407,411-414,422,436,457$, $459,446,466 \mathrm{f}$.

- Dkm. (1911) 422-424

- Dkm. (1921, Waterloo Place) 422

Église de la Madeleine 127

Eiffelturm 295

Elcho, Lord 139

Elizabeth I., Kg.in v. England 417

Elsaß 478-480, 533, 689 (Allegorie) 372

Elsaß-Lothringen 355,483

Elysée-Palast 348

Emerton, J.A. 182

Esher, Reginald B.B., $2^{\mathrm{d}}$ Viscount $413 \mathrm{f}$.

Esterhazy, Marie Charles F. Walsin 484

Eulenburg, Friedrich Gf. v. 486

Euler, Carl 490

Euston Square 599f.

Fabre, Ferdinand

- Dkm. (1903 v. Marqueste) 610

Fallières, Armand 348, 351, 446f., 476f., 580

Farrar, Frederic W. 567

Faubourg Saint-Antoine 283

Faure, Felix 107

Fawcett, Henry 513, 515

- Dkm. (1893) 514, 532

Fehrbellin 170, 202

Ferry, Jules 160f., 355, 361f., 364, 451

- Dkm. (1910) 107, 347, 354, 428f., 431, $480,675 f, 690$ 
Fischer, Emil Hermann 664f,, 669

Fischer, Kuno 591

Floquet, Charles 106f., 159, 280, 298, 344f., 348-350

- Dkm. (1909) 107, 348-350, 429-431, 449,676

Foerster, Wilhelm 306, 594

Fontane, Theodor

- Dkm. (1910) 624, 639, 696

Forest 44If.

Forster, William Edward

- Dkm. (1890) 117

Fowler, Henry $567 \mathrm{f}$.

Fox, Charles James 17, 288

- Dkm. (1816) 138, 288

France, Anatole 645

France-Allegorie 268, 271, 342, 544

François I., Kg. v. Frankreich

- Dkm.-projekt (1852/3) 148

Frankfurt 113

Franklin, John, Sir 255

- Dkm. (1866) 464, 667

Frere, Bartle Sir $457-459$

- Dkm. (1888) 457, 462, 468f., 472, 688

Fresneau, Armand 291

Freytag, Gustav 384f.

Friedrich II., Kg. v. Preußen 165, 207, 234

- Dkm. (1851) 87, 91, 163-166, 168f., 186f., 199, 207, 372, 561, 621, 683

Friedrich III., Kg. v. Preußen 384f., 394

- Denkmäler 371, 393, 434, 684

- Dkm. (1892) 371, 393f., 435

- Dkm. (1903) 394f.

- Dkm. (1904) 90, 395-397, 454

Friedrich Leopold, Prinz v. Preußen 563, 621

Friedrich Wilhelm, Kronprinz d. Dt. Reiches und v. Preußen 561

Friedrich Wilhelm v. Brandenburg, Großer Kfst.

- Dkm. (1703) 169, 561

Friedrich Wilhelm, Gf. v. Brandenburg

- Dkm. (1862) 203, $453 \mathrm{f}$.

Friedrich Wilhelm III., Kg. v. Preußen 87f., 164, 187, 204, 248, 374f., 648

- Dkm. (1849) 87, 110, 162, 186, 648

- Dkm. (1871) 88f., 371-374, 390, 425, 428, 432-434, 455, 493, 617, 672, 679

Friedrich Wilhelm IV., Kg. v. Preußen 87f., 90, 165f., 168f., 187, 199f., 203$207,237,247,377 f$. , 491, 591
- Dkm. (1886) 371, 377f., 433

Friedrichshain $130,135,145,156,165$, $167,198,303 f$., 332, 384, 388, 430

Friedrichstraße 487

Galilei, Galileo 585

- Dkm.-projekt (1898/1900) 587, 605f.

Gambetta, Léon 159f., 277, 281, 342$345,354,480,684$

- Dkm. (1888) 107, 342, 345f., 425 429f., 437, 470, 673, 684

Gardiner, Samuel R. 322

Garibaldi, Giuseppe

- Dkm. (1907) 444-447, 468, 479, 687

Gamier, Francis 450

- Dkm. (1898) 450-452, 471, 687

Garrett, Millicent 514

Gauthier, Théophile 124f.

Gendarmenmarkt 454, 614, 616f.

George I., Kg. v. England

- Dkm. (1748) 405 Anm. 323, 628

George III., Kg. v. England 46

- Dkm. (1838) 17 Anm. 15, 405 Anm. 323

George IV., Kg. v. England

- Dkm. (1843) 405 Anm. 323

George V., Kg. v. England 413-415, 417, 427,673

Gerlach, Andreas Christian

- Dkm. (1890) 593f., 598, 605, 695

Germania 30, 41, 372, 381

- ephemere Denkmäler $(1871,1897) 372$, $379 f$.

Gladstone, Herbert 103, 320

Gladstone, William Ewart 140, 225, 459$463,512,527,530,536,636$

- Dkm. (1905) 117, 509-511, 530, 690

Gneisenau, August W. A. Neidhardt v.

- Dkm. (1855) 204-207, 234, 239

Gneist 493

Goblet, René 583

Godard, Benjamin

- Dkm. (1906, Square Lamartine) 610

Godwin 177

Goethe, Johann Wolfgang v.

- Dkm. (1880) 112, 613-615, 620f., $638-640,696$

Gordon, James, Sir 460-462, 471

- Dkm. (1888) 117, 328, 451, 459f., $462 \mathrm{f} ., 469,688,690$

Goßler, Gustav v. 589

Gottschall, Rudolf $618 \mathrm{f}$. 
Granville, George Leveson-Gower, $2^{\text {nd }}$ Earl of 518

Grévy, Jules 361

Große Friedrichstraße 560

Grunewald 398, 403

Guards Memorial s. Crimea Guards Memorial

Guildhall, London 179

Guise, François I. de Lorraine, Duc de 557

Gutenberg, Johannes

- Dkm. (1851, Imprimérie nationale) 584

Guyot, Yves 552

Hahncke, Wilhelm v. 467

Halifax, Charles Wood, $1^{\text {st }}$ Viscount 225

Hallesches Tor 487

Hamburg 113

Hamel, Ernest 287f.

Hamilton, Edward 527

Hamilton-Gardens 630

Hammersmith Road 465

Hanoi 451

Hanover Square 139, 631

Harcourt, William 416

Hardenberg, Karl A., Freiherr v. 249, 497f., 649

- Dkm. (1907) 486, 496, 498, 531, 534

Hardwicke, Earl of 321

Hargues, Eugène d' 56lf.

Harkort, Christian 111

Harmening, Ernst 500

Hasenheide 486, 488

Haslam, Alfred Seal, Sir 410

Haussmann, Eugène 191

Havard, Oscard $481 \mathrm{f}$.

Havelock, Henry, Sir

- Dkm. (1861) 224, 234, 253, 686

Havin, Léonor 550

Hecker, Friedrich 144

Hegel, Georg Friedrich

- Dkm. (1871) 613, 639

Heinrich VIII., Kg. v. England 566

Helmholtz, Hermann v. 114

- Dkm. (1899) 114f., 594, 603, 606, 695

Henri IV., Kg. v. Frankreich 16, 57

Henricy 583

Herbert, Sidney, Baron of Lea 23 If.

- Dkm. (1867) 116, 229-233, 237, 239, $665 f$., 686

Hercules 41

Hermann 490
Hermann-Dkm. 29, 54

Hessische Straße 664

Highgate 515

Hill, Rowland, Sir 600

Hindenburg, Paul v. Beneckendorff u. v.

- Dkm. (1915) 660-662

Hohenlohe-Schillingsfürst, Chlodwig, Fst. zu 379

Holborn 179

Hopper, Thomas 212

Horse Guards Parade 466, 512

Hôtel de Ville 262f., 362, 367, 555

Houghton, Richard Monckton Milnes, $1^{\text {st }}$ Baron 631

Houses of Parliament 145, 209, 321

Hovelaque, Abel 288

Hugo, Victor 106, 265, 362, 551f., 558, 612

- Dkm. (1902) 611, 638f.

Humboldt, Alexander v. 91, 198, 590, 614

- Dkm. (1883) 91, 114, 588-594, 603605, 694f.

Humboldt, Wilhelm v. 91, 589f.

- Dkm. (1883) 91, 588-594, 603-605, $694 f$.

Hume, Joseph 219

Huskisson, William

- Dkm. (1848) 136, 145, 674

Hyde Park (Comer) 98f., 139, 180, 188f., $214,217 f$., 220f., 412, 423, 465, 630, 632

Île de Cygnes 359

Indien $223-225,227,234,458$

Indochina 452

Inglis, Robert, Sir 219

Inselstraße 500

Institut de France 360

Invalidendom 151, 221

Invalidenfriedhof $197 \mathrm{f}$.

Invalidensäule 64, 111, 168, 198-204, 233f., 237-239, 686

Ireland, John, Dean of Westminster 630

Irland $212,224,465,675$

Jacobikirchhof 498f, 504

Jahn, Friedrich Ludwig 488f.

- Dkm. (1872) 92, 112, 486, 488-490, $531,534,691$

James II., Kg. v. England und Schottland

- Dkm. (1685) 405 Anm. 323 
Jardin d'Acclimatation 246

Jardin de l'Infante 448

Jardin des Plantes 646

Jardin des Tuileries 81, 324f., $350 \mathrm{f}$.

Jardin du Luxembourg 81, 241, 477, 610, 646

Jardin du Musée Carnevalet 292

Jardins Denfert Rocherau 301, 376

Jaucourt, Jean François 556

Jeanne d'Arc 541-549

- Denkmäler 482, 550, 552, 557, 571, 573f., 576, 653, 659, 676, 693

- Dkm. (1874) 346f., 541-548, 573

- Dkm. (1891) 547

- Dkm. (1900) 547-549

Jenner, Edward

- Dkm. (1858) 116, 253-5, 257, 259

Joachim II., Kfst. v. Brandenburg

- Dkm. (1889) 111, 371, 391-393, 435

Joffrin, Jules 108, 274f., 361

John Bull 45, 51

Johnston, William 319

Jourdan, Louis 194-196

Julisäule $127,245,362 f$.

Jung, Georg 131

Kaiser-Wilhelmstraße 652

Kalbaum 170

Kameke, Georg v. 453

Karlsplatz 505

Keith, Jakob

- Dkm. (um 1859) 206f.

Kemperplatz 389

Kensington $176,254,407 f$., 411, 415, 426, 434f., 569

Kensington Gardens bzw. Palace 326, 406, 411

Kerst $488-490$

Khartum 459-461, 463f.

Kirschner, Martin $502 \mathrm{f}$.

Kléber, Jean-Baptiste 479

Knightsbridge 465

Koch, Robert

- Dkm. (1916) 663

Köln 113

Kölner Dom 172

Königsplatz 252, 389, 398f., 403f., 452, 454f., 661

Köpenick(er Straße) 500, 503

Koser, Alfred Reinhold 626

Kreuzberg 134

- Dkm. (1821) 134, 208
Kronprinzenpalais 247

Krupp, Alfred

- Dkm. (1899) 588, 595-597, 604, 606f., 695

Lacour, Edmond 354

Lake, Dean of Durham 569

Lalance, Jean 478

Lamartine, Alphonse de 245, 298f., 303

- Dkm. (1886) 295, 297

Lambeth $514 f$.

Langerhans, Paul 305, 307

Lansdowne, Henry Petty-FitzMaurice, $5^{\text {th }}$ Marquess of 512

Larrey, Dominique

- Dkm. (1850) 105, 24l-244, 254, 256$258,584,694$

Larroumet, Gustave 447

Lassalle, Ferdinand

- ephemeres Innen-Dkm. (1892) 598

Laussedat, Aimé 582

Lavoisier, Antoine Laurent de

- Dkm. (1900) 108, 585

Lawrence, John, Sir

- Dkm. (1882) 226, 667

Lawson, Wilfried 319

- Dkm. (1909) 514, 517, 532, 675

Le Verrier, Urbain

- Dkm. (1889) 110, 583f.

Leblanc, Nicolas

- Dkm. (1887) 108, 581f., 603

Ledru-Rollin, Alexandre 301, 303

- Dkm. (1885) 83, 106, 296f., 299

Lefevre 286

Leibniz, Gottfried Wilhelm 585

Leicester Square 628

Leipzig (Völkerschlacht) 163, 202, 247

Leipziger Platz 203, 453

Leipziger Straße 492

Lennéstraße 621

Leopold I., Fst. v. Anhalt-Dessau

- Dkm. (um 1859) 206f.

Lépine, Louis Jean-Baptiste 369

Leprévost, Maurice 550

Leroy-de-Saint-Amaud, Achille A.J. $192 f$.

Lessing, Gotthold Ephraim

- Dkm. (1890) 112f., 613-615, 621-623, 638-640, 696

Levetzow, Albert v. 399

Lewis 14lf.

Liberté-Allegorie 270, 289, 359

Lichterfelde 207 
Liebknecht, Karl 507

Lille 548

Linden-Allee $s$. Unter den Linden

Lissagaray, Prosper Olivier 270

Lloyd George, David 419

Lockroy, Édouard 300

Longuet, Charles 445

Lothringen $344,364,372$

Loubet, Émile 284, 351, 367f., 586

Louis-Philippe I., Kg. v. Frankreich $151 f$.

Louise, Duchess of Argyll, engl. Prinzessin $407,411,515$

Louvre $129,147,191,343,359,448$

Lucipia, Louis Adrien 368

Ludwig XIV., Kg. v. Frankreich 561

Luise, Königin v. Preußen 162f., 170, $187,380,391,647 \mathrm{f}$.

- Denkmäler 648-650, 652, 654, 662

- Dkm. (mit Friedrich Wilhelm III. 1809) 170,390

- Dkm. (1858) 170-172, 185, 202, 646, 696

- Dkm. (1880) 90, 11lf., 646-650

- provisorisches Dkm. (1897) 380

Luiseninsel 390,646

Luisenplatz 170,663

Luisenstraße 197, 505, 593

Lustgarten 205, 372, 432, 455, 617

Luther, Martin 16, 560, 564

- Dkm. (1895) 562f., 565, 577, 692

Macaulay, Thomas B. 322

Macé, Jean

- Dkm. (1900) 107, 558

MacMahon, Edme, Gf. v. 272, 341

Madier de Montjau, Noël 297

Maginot, André 659

Mahias, J. 160

Mahy, François de $302 \mathrm{f}$.

Mall, The 101, 329f., 601, 667

Manchester 140

Manners, John, Lord 253, 525

Manning, Henry E. 117

Manteuffel, Otto v. 168, 491

Marat, Jean-Paul 294

- Dkm. (1887) 258, 288, 290-292, 333, 337, 677

Marcère, Émile de $357 f$.

Maret, Henri 256f., 345

Marianne 30,41

Marie-Louise, Kaiserin v. Frankreich,

Gemahlin Napoleons I. 152
Mark Brandenburg 170

Marlborough House 464

Marseille 160

Marsfeld 369, 431, 442

Martin, Georges 275

Martin, Marius 282

Märzgefallenen, Dkm. für die 93, 130 $135,145,155,189 f$., 197, 539, 677, 680-683

Mathé 363f.

Mayer, James W. 568f.

McGrigor, James Sir 254-5

- Dkm. (1865) 97, 116, 254-5, 258, 694

Meding, August Friedrich Wilhelm v. $248 f$.

Meissonier, Ernest

- Dkm. (1895) 110, 448f., 482

Melbourne, William, Lord 98

Merriman, John J. 118

Metropolitan (Police) District 95f.

Michel, Louise 300

- Dkm.projekt (1907) 643f., 653

Michelet, Jules 123, 280, 542

Michelet, Paul 506

Michelin 553

Mill, John Stuart

- Dkm. (1878) 104, 119, 513f., 536

Millerand, Alexandre 352

Molesworth, William, Sir 138

Moltke, Helmut Gf. v.

- Dkm. (1905) 455, 493, 661

Mommsen, Theodor 500

Monbijouplatz 623

Moncey, Jeannot de 196, 235

Mond, Alfred, Sir 329

Montmartre 294

"Monument commémorant le 24 Février 1848«(Dkm.-projekt 1848) 128

"Monument de la Femme Française" (Dkm.-projekt 1909) 644f., 653

„Monument Henry“ (1898/99) 484-486, 539

Morley, John 316, 510

Morpeth, George W.F. Howard, $7^{\text {th }}$ Earl of Carlisle, Lord 98, 218, 220

Mosse, Rudolf 622

Mouchez, Ernest Amedée B. 584

Moulton, William 567

Müffling, Karl v. 166

Müggelberge 403f.

München 9

Munckel, Carl August 310 
Mundella, Anthony John 319

Mur des Fédérés 64, 108, 272f., 278f., $281,331,334,361,677,682$

Murchison, Sir R. 255

Museumsstraße 395

Napier, Charles James

- Dkm. (1856) 223f., 234, 237, 686, 688

Napier, Robert Comelis

- Dkm. (1891) 424, 464-466, 472

Napoleon I. Bonaparte, Ks. v. Frankreich $15,43,105,147,149,153,155,160$, $193,196,221,241,243-4,258,262$, 270, 374f., 443, 449, 481-483, 495f., 534, 537f., 564, 612, 635, 647, 680, 683,685 [s. auch Vendômesäule]

- Dkm. (1810, Vendômesäule) 151, 153, 270

- Dkm. (1833 »Petit Caporal«, Vendômesäule; 1863 Courbevoie) $38,151-154$, 195 (1871 Sturz, um 1912 Cour des Invalides) $269 \mathrm{f}$., $442 \mathrm{f}$.

- Dkm. (1863) 151-154, 186, 238, 262, 269f., 544, 550

- ephemeres Dkm. (1852) 148

- Reiterdenkmalprojekte (um 1860/1862) 147

Napoleon III., Ks. v. Frankreich 81-83, $105,147-155,157,159,185 f ., 190-$ $192,195,226,230,235,237,242,262$, $301,334,362,374,441 f$., $444 f$., 612 , 683

- Dkm.-projekte 148

National Gallery 667

Nefftzer, Auguste 154f., 157f.

Nelson, Horatio, Viscount 49, 210, 212, 461

- Dkm. (1843) 97f., 115, 208-216, 22lf., 235f., 253, 675, 685

- Dkm. (1805, Dublin) 212

- Dkm. (1807, Edinburgh) 212

Neue Wache 91, 168, 204, 207, 234, 238

Neuer Markt 562

Neuville, Alphonse de

- Dkm. (1889) 447f., 482

Newman, John Henry

- Dkm. (1896) 569-572, 576, 693

Newton, Isaac 585

- Dkm.-projekt (1895) 587, 605f.

Ney, Michel 193, 233

- Dkm. (1853) 82f., 191-196, 233, 235, 238f., 242, 257, 479, 685
Nicolaikirche 391

Niederwalddenkmal 30

Nightingale, Florence 232

- Dkm. (1915) 665-668, 698

Nizza 444

Northcote, Stafford, Sir 523-525

Notre-Dame 548

Observatoire $83,450,583 \mathrm{f}$.

Opernplatz 204

Oranienburg 170f., 185, 654

Oranienstraße 498f.

Oranje-Freistaat 458

Orléans 545,548

Oustry, Louis 363

Outram, James, Sir

- Dkm. (1871) 117, 225f., 234, 237, 686

Oxford 570

Painlevé, Paul 685f.

Pakington, John, Sir 255

Palace Yard (New) 102, 139, 330

Pall Mall 229, 464

Palmerston, $3^{\text {rd }}$ Viscount $175,219,224$, 229f., $508 f$.

- Dkm. (1876) 102, 140, 508, 531, 536, 690

Pantheon (Sainte Geneviève) 37, 255, $289,362,299,332,553,611$

Parc des Buttes-Chaumont 292

Parc Monceau 610

Parc Montsouris 291

Pariser Platz 168

Parliament Square 138, 140, 320, 508, 519,523

Parmentier, Antoine

- Dkm. (1866) 106, 245

Pasteur, Louis

- Dkm. (1904) 586, 603, 605

Péan, Jules

- Dkm. (1909) 580

Peel, Robert, Sir, $2^{\text {nd }}$ Baronet $137-139$, 219,229

- Dkm. (1852, Westminster Abbey) 137

- Dkm. (1855) 102, 116, 137, 145

- Dkm. (1876 bzw. 1866) 138-140, 145

Peel, William Robert $510 f$.

Pelletan, Camille 300

Père Lachaise 64, 149, 267, 272, 274, 276, 278, 280f., 332

Perls, Arnold 305

Petit, Jean Martin 105, 241, 243 
Peyrat, Jean-Alphonse $157 \mathrm{f}$.

Picadilly Circus 518

Picquard, Georges 476, 478, 612

Pinel, Philippe

- Dkm. (1885) 108, 579

Piper, Ferdinand 621

Pitt, William (the Younger)

- Dkm. (1778, Westminster Abbey) 288f

- Dkm. (1831) 17, 139

Place André-Tardieu 482

Place Carrousel 34If., 346

Place de Breteuil 586

Place de Clichy 196

Place de Guatemala 347

Place de l'Étoile/Triumphbogen 149, 153 , 440

Place de la Bastille 127, 244f., 283, 362 , 449

Place de la Concorde $346,358,362,548$

Place de la Madeleine 585

Place de la Nation (Place du Trône) 289, $365,367,548$

Place de la République $360-362,440$

Place des Abbesses 644

Place des Pyramides 346f., 542, 546-549, 571,573

Place des Victoires 16

Place du Palais Royal 610

Place Malesherbes 609

Place Maubert 109, 554f.

Place Monge 299

Place Montrouge 560

Place Napoléon (Louvre) 147

Place Saint-Germain-des-Prés 268

Place St.-Augustin 547f.

Place Vendôme 15, 263, 267f.

Place Victor Hugo 611

Place Voltaire 296

Place Wagram 447

Plunket, David Robert 459, 463

Poincaré, Raymond 448f., 657

Pommer-Esche, Johann v. 251

Ponsonby, Henry F., Sir 412

Pont-de-Grenelle 289

Pont de Neuilly 442

Potter, Eugène 277

Poubelle, Eugène René 275, $291 \mathrm{f}$.

Pressensé, Francis de 612, 644

Primerose Hill 629

Prince of Wales-Avenue 629

Punjab 464

Puy, D.J. du $127 f$.
Pyat, Félix 267

Quai Conti 360

Quai de Valmy 366

Quai Malaquais 549, 552

Quillard, Pierre 484-486

Raffet, Denis-Auguste

- Dkm. (1893) 448, 482

Railton, William 98

Ranke, Leopold v. 88

Raspail, François de 301

- Dkm. (1889) 109, 289, 295f., 338

Recke, Horst v. d. 93

Reformationsplatz 391

Regent's Park 629

Regnault, Henri

- Dkm. (1876) 439f., $468 f$.

Reichenbach, Eduard Graf v. 132

Reichstag 27, 379, 404, 456

Reinach, Josef 484

République

- Denkmäler 124f., 127-9, 142f., 356, $376,425 f$., $435,532 f$., $653,672-675$, $677,684,689$

- (nicht öffentliches) Dkm. (1848) 124f., 126, 244, 359, 370, 43 If., 680 f.

- Dkm. (1878) 357-359, 369

- Dkm. (1880) 359f., 370, 432

- Dkm. (1883) 106, 360-365, 370, 429, 440,684

- Dkm. (1899, "Triomphe de la R. () 106, $365-370,426,432,480,675,679,684$, 690

Réveillon, Tony 300

Reyher, Karl Friedrich Wilhelm v. 163

Richter 133

Richter, Eugen 309

- Dkm.-projekt (1909) 506f.

Rivet, Gustave 446

Roberts, Frederick, $1^{\text {st }}$ Earl of 465

Robertson, Andrew 211-213

Robespierre, Maximilien de

- Dkm.-projekt (1909) 268, 288, 294f., 333

Roche, Adrien 128

Rochefort, Henri 273

Rochow, v. 166

Roon, Albrecht Gf. v. 456

- Dkm. (1904) 454, 456, 471, 687

Roquefeuille de 547

Rosebery, Archibald Philip Primrose, 
$5^{\text {th }}$ Earl of $101,103,315,319,321-$ $325,636 f$.

Rousseau, Jean-Jacques

- Dkm. (1889) 107, 287-290, 332, 337

Roux $243 f$.

Royal Courts of Justice 509

Royal Exchange 136, 600

Royer, Clémence

- Dkm.projekt (1912/1914) 645f., 653

Rue de la Santé 580

Rue de Rivoli 556

Rue du Faubourg-Saint-Antoine 158

Rue du Faubourg-du-Temple 366

Rue Lamarck 558

Ruskin, Fabian John 463

Russel, John, Lord $219 \mathrm{f}$.

Rußland 202, 229f.

Sabran-Pontevès, Guillaume, Comte de $543 \mathrm{f}$

Sachs 308,499

Sacré-Cœur 294, 558, 693

Saint-Just, Louis de 294

Salisbury, Robert Gascoigne-Cecil, $3^{\text {rd }}$ Marquess of $100,103,117,325$, $457,466,512,523,524 \mathrm{f}$.

Sankt Helena 151, 153, 195

Sarrelouis 193

Savoyen 444

Schamhorst, Gerhard v.

- Dkm. (1822) 91, 168, 204

Scheurer-Kestner, Auguste $477 \mathrm{f}$.

- Dkm. (1908) 475, 477-480, 532f., 688f.

Schiller, Friedrich

- Dkm. (1871) 112, 613-621, 638-640, 696

Schinkelplatz 247

Schlenk, Wilhelm 664

Schloßbrücke 206, 234, 379

Schmidt, Erich 621

Schomberg-McDonnell 412

Schönhauser Allee 596

Schulze-Delitzsch, Hermann 502f, $505 \mathrm{f}$.

- Dkm. (1899) 92, 115, 486, 500f., 504, 531,691

Schwerin, Kurt Christoph v.

- Dkm. (um 1859) $206 f$

Scott, George Gilbert 100, 183, 228

Scott, Robert Falcon 667-669

Scott, Walter 637

Selves, Justin Germain C. de 368
Senefelder, Alois

- Dkm. (1896) 588, 598f., 604, 606, $675 f$.

Servaes, August 595f.

Servet, Michel

- Dkm. (1908) 554, 560, 576, 693

Seurre, Émile 442

Seydel, Karl $617 f$.

Seydlitz, Friedrich Wilhelm v.

- Dkm. (um 1859) 206f.

Shaftesbury, Lord $518 \mathrm{f}$.

- Dkm. (1893) 514, 518, 536

Shakespeare, William

- Dkm.-projekte $(1851,1863)$ 628-630, 638,640

- Dkm. (1874 Leicester Square) 627f., 696,640

- Dkm. (1888 Avenue Messine) 609

Shaw-Lefevre, George John 328

Siegesallee 389f., 394

Siegessäule $452,454,456,487$

Siemens, Werner v. 114,594

- Dkm. (1899) 114, 588, 595, 597, 604, 606f., 695

Simon, Jules $268,270,290,439,448$

- Dkm. (1903) 347, 430

Simoneau 282

Singer, Paul 305-307

Soitoux, Jean François 124, 126, 143 , 359,370

Spandau 111, 371, 391, 393, 453

Spinola 306

Spuller, Eugène 106, $343 \mathrm{f}$.

Square Cambronne 444

Square du Temple 481

Square Marcelin Berthelot 583

Square Marie-Curie 579

St. Clement Dane's Church 517

St. James's Palace/Street 226, 412, 414, 632

St. Martin's Place 667

St. Pancras 97, 140f.

St. Paul's Cathedral 212, 217, 651

StadtschloB, Schloßfreiheit 27, 89, 91, 131, 205, 306, 371f., 379-381, 387, $389,393 f, 425,429,487$

Staël, A. L. Germaine de

- Dkm.-projekt (1909) 644

Stanley, Arthur Penrhyn 566

Stein, Karl Freiherr vom $495 \mathrm{f}$.

- Dkm. (1875) 90f., 486, 491-494, 496, $531,534,691$ 
Stephenson, George

- Dkm. (1854) 599

Stephenson, Robert

- Dkm. (1871) 560

Strasbourg

- Dkm. (1836) 346, 362, 450, 548

Strathnaim, Baron

- Dkm. (1895) 465f., 469, 688

Sydow, Karl L.A. 131, 617

\section{Tannenberg 660}

Technische Hochschule (Charlottenburg) 595

Tempelhofer Chaussee 372

Temple de l'Oratoire 556

Temple of British Worthies, Stowe 17

Temple of Liberty, Wobum 17

Tenot, Eugène 158

Texier, Edmond 154f.

Thaer, Albrecht 248

- Dkm. (1860) 247f., 250, 256, 258f., 426, 675, 694

Thames Embankment 225

Thibaudin, Jean 443

Thiers, Adolphe 272, 277, 281, 316, 347, 442-444

Thomas, Frédéric 270

Thornycroft, Hamo 316

Thorwaldsen, Berthel 631

Tierärztliche Hochschule 593

Tiergarten 162, 170, 506, 620f., 624f., $646,648,650$

Tilsit 455

Tonking 451

Tooting Broadway 422

Tour 344

Trafalgar Square 97, 115, 140, 209, 214 $216,222-224,232,235,237,253-5$, 259,459

Transvaal 458, 527

Trarieux, Ludovic $367,476,612,688 f$.

- Dkm. (1907) 475-477, 480, 532f.

Treitschke, Heinrich v. 564f., 649

- Dkm. (1909) 626f., 696

Trouillot, Georges 452

Tuaillon, Louis 662

Tuilerien 147, 262, 34lf., 346, 475

Tunesien 452

Tupper, Charles, Sir 118

Tuyen-Quan 449

Tyndale, William

- Dkm. (1884) 566f., 577, 692
Tyndall, John $633 \mathrm{f}$.

Unter den Linden 91, 163, 165f., 168, 204f., 372, 394, 456, 487, 561, 588, $590,592,603,614,621,650$

Vaillant, Éduard 276f., 644

Val-de-Grâce 105, 241

Vauquelin, Nicolas

- Dkm. (um 1875) 106, $245 f$.

Vauxhall Park 514

Vendôme-Säule [s. Napoleon I.] 38, 151154, 186, 195, 550 (Sturz 1871) 262268, 330, 442f., 677, 681, (Wiedererrichtung 1875) 267-272, 331, 334, 543f., 573, 68 If.

Vercingetorix 29, 54

Versailles 128, 262, 455

Vicaire, Gabriel

- Dkm. (1902, Jardin du Luxembourg) 610

Victoria, Königin v. Großbritannien 46, 98-102, 119, 172, 176, 182-184, 187, $212,218,237,329,405 f$., $409,417 f$., $422,426 f$., $436,525,532,578,643,651$

- Denkmäler 405, 425, 673, 679, 685

- Dkm. (1893) 118, 406-410, 415, 425428,435

- Dkm. (1896) 410f., 435

- Dkm. (1904) 41 lf.

- Dkm. (1911) 100, 412-422, 425f., 428, 435f., 601,673

Victoria Embankment 104, 410, 457, 513, 566,635

Viktoria, Kaiserin

- Dkm. (1903) $394 f$.

Viktoria-Allegorie 648

Vincennes 197

Virchow, Rudolf 114, 499-502, 506, 589 f.

- Dkm. (1910) 93f., 505, 531, 553, 691, 695

Vivian, Herbert 327

Viviani, René 645

Voltaire 84, 287-289, 541, 544f., 551, $556,558,572,574,693$

- Dkm. (1885) 549f,, 552f.

- Dkm. (1887) 553f.

Vorbe $587 f$.

Waddington, William 110

Wagner, Richard 
- Dkm. (1903) 625f., 640, 696

Waldeck, Franz

- Dkm. (1890) 115, 486, 498-500, 502, $531,535,676,691$

Waldeck-Rousseau, Pierre 284, 350-353, $356,365,440 f ., 611$

- Dkm. (1910) 107, 354f., 429, 431, 480, 690

Walhalla 145,172

Wandsworth 422

War Office 466

Waterloo (Belle Alliance) 170, 193, 202, 205, 210, 219, 234-237

Waterloo Place 218, 222, 224, 229, 236f., 255, 422, 424, 464f., 665, 667

Waterlow, Sidney, Sir 516

- Dkm. (1900) 514f.

Waterlow Park 515

Webb, Aston 60I

Wellington, Arthur Wellesley, Duke of $46,99,115,136,174,219-221,236$, $254,461,466,685 f$.

- Dkm. (1822 »Achilles« v. R. Westmacott, Hyde Park) 212, 217

- Dkm. (1844) 217

- Dkm. (1846) 98f., 139, 212, 217-222, 236, 239, 465 (Entfernung 1883) 677

Wenckstern, Adolph von 497

Wermuth, Adolf $662 \mathrm{f}$.

Wesley, John

- Dkm. (1891) 567-569, 572, 577, 692

Westminster 316, 320f., 514, 572, 576

Westminster Abbey 44, 137, 212, 288 , $321,509,570$

Westminster Palace 102, 311, 321

Westphalen, Ferdinand v. 491f.

Whitehall (Place) 225, 228, 330, 466, 512

Wilberforce, Samuel, Bf. v. Oxford $230 f$.

Wilhelm, Karl 455

Wilhelm I., Kg. v. Preußen, dt. Kaiser 28, 29, 88-92, 119f., 132, 206-208, 234, $238,250-252,256,265,374,377,383$, $387,394,396,456,616,646-648,650$

- Denkmäler 308, 371, 380, 428, 672, $674,678,684$

- Dkm. (1897) 89, 306, 379-389, 393, $425,428,430,433,672,675,679$

- Dkm. (1902) 389 Anm. 242

- Dkm. (1904) 390f.

Wilhelm II., dt. Kaiser 89f, 92, 94, 113 , 119f., 265, 374, 379, 381f., 389f., 393395 , 398f., 413f., 427, 436, 455, 467,
$501,563,589,594,597 f$ f, $638 f ., 650$, 654, 672 (Kronprinz) 620, 622

Wilhelm (William) III., Statthalter der

Niederlande, Kg. v. England

- Dkm. (1808) 405 Anm. 323

- Dkm. (1907) 326, 371, 617

Wilhelmplatz 16, 168, 205f., 234, $238 f$.

William IV., Kg. v. England

- Dkm. (1844) 405 Anm. 323

Williamson, G. A. 424

Winchester 324f.

Winterfeld, Hans Karl v.

- Dkm. (um 1859) $206 f$.

Woolwich 232

Wrangel, Friedrich v. 454

- Dkm. (1880) 90, 203f., 453, 471, 687

Wyatt, Matthew C. 98

York, Duke of

- Dkm. (1834) 17, 228, 255, 464, 667

Yorck v. Wartenburg, Hans D. L.

- Dkm. (1855) 204-207, 234, 239, 374

Zelle, Robert 93, 307, 564

Zeughaus 204, 207, 250

Zieten, Hans Joachim v.

- Dkm. (um 1859) $206 f$.

Zola, Émile 367, 478, 612

- Dkm. (1924) 612, 639 


